\title{
Mesures de houles partiellement stationnaires en zones cotière et littorale en présence de courant
}

\author{
Déborah Drevard, Vincent Rey et Philippe Fraunié
}

\author{
LSEET-LEPI, Université du Sud-Toulon Var, BP 20132, 83957 La \\ Garde cedex
}

\section{Résumé :}

Des expériences en bassin ont été menées pour des houles régulières et irrégulières en présence de courant, où coefficients de réflexion calculés à partir des données synchrones de vitesses horizontale et verticale ont été comparés avec succès à la méthode classique à trois sondes. Des mesures de réflexion en zone de shoaling ou de déferlement à partir de données synchrones de la vitesse horizontale et de la vitesse verticale ou de la pression sont ensuite présentées. On montre que les données synchrones de vitesse horizontale et de pression donnent de bons résultats quelle que soit la profondeur d'immersion de l'appareil alors que la méthode basée sur les mesures synchrones des vitesses horizontale et verticale nécessite une immersion de l'appareil à faible distance de la surface.

Mots-clefs : houle / spectre / réflexion / littoral / mesures

\section{Abstract :}

In the present work, methods for partially standing wave measurements in the presence of current by use of coincident measurements of both horizontal velocity and pressure or vertical velocity are described. Taking advantage of experiments carried out in an ocean wave basin for both regular and irregular waves in the presence of current, comparisons are made for reflections calculated from either coincident horizontal and vertical velocities or three gauge methods. Application to field measurements outside and in the breaking zone are then presented. It is shown that the use of coincident horizontal velocity and pressure, or coincident horizontal and vertical velocities far from the bottom, give relevant information concerning partially standing waves nearshore.

\section{$\underline{\text { 1-Introduction }}$}

La houle au large est caractérisée par des grandeurs significatives telles que la hauteur $\mathrm{H}$, la période $\mathrm{T}$ et la direction moyenne $\theta$. A l'approche du littoral, la propagation des vagues est modifiée par effets bathymétriques et une partie de l'énergie peut être partiellement réfléchie par des pentes raides, des brises-lames immergés, le haut des plages. Les instruments de mesure immergés généralement déployés en zone littorale permettent de mesurer les champs de vitesse et/ou de pression et sont basés sur des dispositifs acoustiques ou électromagnétiques.

Les plages peuvent être classifiées comme dissipatives, réflectives ou intermédiaires $^{9}$, cependant la réflexion est rarement mesurée. Walton ${ }^{8}$ a mesuré la 
réflexion des vagues par une plage au moyen de données synchrones de vitesse horizontale et de pression enregistrées en un point donné. Cette technique a été récemment appliquée dans des expériences en bassin à partir de données synchrones de vitesses horizontale et verticale ${ }^{5}$. En zone côtière, la marée et le déferlement induisent du courant, pouvant être suffisamment fort pour avoir de l'influence sur la propagation des vagues. En présence d'un courant donné, les effets Doppler doivent être pris en compte afin de séparer les ondes incidente et réfléchie. Ce principe a été utilisé lors d'expériences en bassin pour des mesures de réflexion de vagues à partir de données synchrones de trois sondes à houle ${ }^{6,7}$.

Dans ce travail, nous avons mesuré la réflexion à partir de données synchrones de vitesses horizontale et verticale ou de pression. Pour cela, nous avons utilisé des instruments PUV mesurant la vitesse horizontale et la pression (S4, système électromagnétique), et PUVW mesurant les vitesses horizontale et verticale et la pression ( $\mathrm{ADV}$, système acoustique). Des expériences en bassin ont permis de comparer les résultats avec des sondes à houle. Des applications in situ hors puis dans la zone de déferlement sont ensuite présentées.

\section{2-Méthode de calcul des caractéristiques d'une onde partiellement réfléchie}

Dans l'hypothèse d'une même direction de propagation pour les ondes incidentes et réfléchies, on se limite au plan xOz. La surface libre $\eta(x, t)$ pour une onde de fréquence $f=\omega / 2 \pi$ résultant de deux ondes se propageant dans la direction $(0 \mathrm{x})$, avec des sens opposés s'écrit:

$\eta(x, t)=a_{i} e^{i\left(\omega t-k^{-} x\right)}+a_{r} e^{i\left(\omega t+k^{+} x+\varphi\right)}$

où $a_{i}$ et $a_{r}$ sont les amplitudes des ondes incidente et réfléchie, et $\varphi$ la phase à l'origine. $k^{ \pm}$correspondent aux nombres d'onde des ondes incidente (propagation vers les $\mathrm{x}>0$ ) et réfléchie, et sont donnés par les relations de dispersion:

$\left(\omega \pm|U| k^{ \pm}\right)^{2}=\left(\sigma^{ \pm}\right)^{2}=g k^{ \pm} \tanh \left(k^{ \pm} h\right)$

avec $\mathrm{h}$ profondeur d'eau.

Le potentiel de vitesses correspondant est de la forme:

$\phi(x, z, t)=\frac{i a_{i} c_{v}^{-}}{k^{-}} e^{i\left(\omega t-k^{-} x\right)}+\frac{i a_{r} c_{v}^{+}}{k^{+}} e^{i\left(\omega t+k^{+} x+\varphi\right)}+U x$

avec $c_{v}^{ \pm}=\frac{\sigma^{ \pm} \cosh \left(K^{ \pm}\right)}{\sinh \left(k^{ \pm} h\right)}$ et $K^{ \pm}(z)=k^{ \pm}(z+h)$, avec z vertical orienté vers le haut.

Le champ de vitesses est alors donné par $\vec{u}(u, w)=\vec{\nabla} \phi$, et la pression par $p=-\rho \frac{\partial \phi}{\partial t}$.

Les amplitudes des ondes incidente et réfléchie $a_{i}$ et $a_{r}$ sont données, à partir de $(\mathrm{u}, \mathrm{w})$ par : 


$$
\begin{aligned}
& a_{i}=C_{u w}\left[\left(s_{v}^{+}|u|\right)^{2}+\left(c_{v}^{+}|w|\right)^{2}+2 s_{v}^{+} c_{v}^{+}|u \|| w \mid \sin \left(\varphi_{w}-\varphi_{u}\right)\right]^{1 / 2} \\
& a_{r}=C_{u w}\left[\left(s_{v}^{-}|u|\right)^{2}+\left(c_{v}^{-}|w|\right)^{2}-2 s_{v}^{-} c_{v}^{-}|u \| w| \sin \left(\varphi_{w}-\varphi_{u}\right)\right]^{1 / 2} \\
& \text { Avec } C_{u w}=s_{v}^{-} c_{v}^{+}+s_{v}^{+} c_{v}^{-} \text {et } s_{v}^{ \pm}=\frac{\sigma^{ \pm} \sinh \left(K^{ \pm}\left(z_{v}\right)\right)}{\sinh \left(k^{ \pm} h\right)} .
\end{aligned}
$$

et à partir de (u,p) par :

$$
\begin{aligned}
& a_{i}=C_{u p}\left[\left(c_{p}^{+}|u|\right)^{2}+\left(c_{v}^{+}|p|\right)^{2}+2 c_{p}^{+} c_{v}^{+}|u \| p| \cos \left(\varphi_{u}-\varphi_{p}\right)\right]^{1 / 2} \\
& a_{r}=C_{u p}\left[\left(c_{p}^{-}|u|\right)^{2}+\left(c_{v}^{-}|p|\right)^{2}-2 c_{p}^{-} c_{v}^{-}|u \| p| \cos \left(\varphi_{u}-\varphi_{p}\right)\right]^{1 / 2} \\
& { }_{\text {avec }} C_{u p}=c_{p}^{-} c_{v}^{+}+c_{p}^{+} c_{v}^{-} \text {et } c_{p}^{ \pm}=\frac{\rho \omega \sigma^{ \pm} \cosh \left(K^{ \pm}\left(z_{p}\right)\right)}{k^{ \pm} \sinh \left(k^{ \pm} h\right)}
\end{aligned}
$$

avec $u=|u| e^{i \varphi_{u}}, w=|w| e^{i \varphi_{w}}, p=|p| e^{i \varphi_{p}}$.

Les mesures des vitesses $\mathrm{u}^{(\mathrm{m})}, \mathrm{v}^{(\mathrm{m})}, \mathrm{w}^{(\mathrm{m})}$ et de la pression $\mathrm{p}^{(\mathrm{m})}$ (où $(\mathrm{m})$ fait référence aux valeurs mesurées) s'effectuent à une profondeur donnée, $z_{v}$ et $z_{p}$ respectivement. Après FFT, on applique à chacune des composantes spectrales de fréquence $\mathrm{f}$ les algorithmes précédents, sachant que la vitesse horizontale est donnée par $\left|u_{h}\right|^{2}=|u|^{2}+|v|^{2}$.

\section{$\underline{3 \text { Validation expérimentale en bassin }}$}

Des mesures de réflexion des vagues sur un fond sinusoïdal en présence de courant ont été réalisées au BGO-FIRST (Var) ${ }^{6}$. Nous avons étudié ici la capacité de l'ADV à mesurer la réflexion de l'onde incidente par le fond sinusoïdal en comparant les résultats aux données de sondes à houle. Les expériences ont été effectuées avec un courant de $0.32 \mathrm{~m} / \mathrm{s}$ dans le sens de propagation de l'onde incidente pour une houle régulière puis irrégulière.

Le coefficient de réflexion pour une houle monochromatique se propageant avec courant est présenté dans la Fig. 1(a) en fonction de la fréquence f. Une réflexion importante est observée théoriquement dans les conditions de résonance de Bragg $^{6}$, autour de $\mathrm{f}=0.52 \mathrm{~Hz}$.

Les résultats des données des sondes à houle et de l'ADV sont généralement en bon accord. Expérimentalement, on observe une réflexion plus faible pour $\mathrm{f}=0.52$ $\mathrm{Hz}$, qui peut s'expliquer par la convergence de la houle au dessus du fond sinusoïdal ${ }^{6}$. 

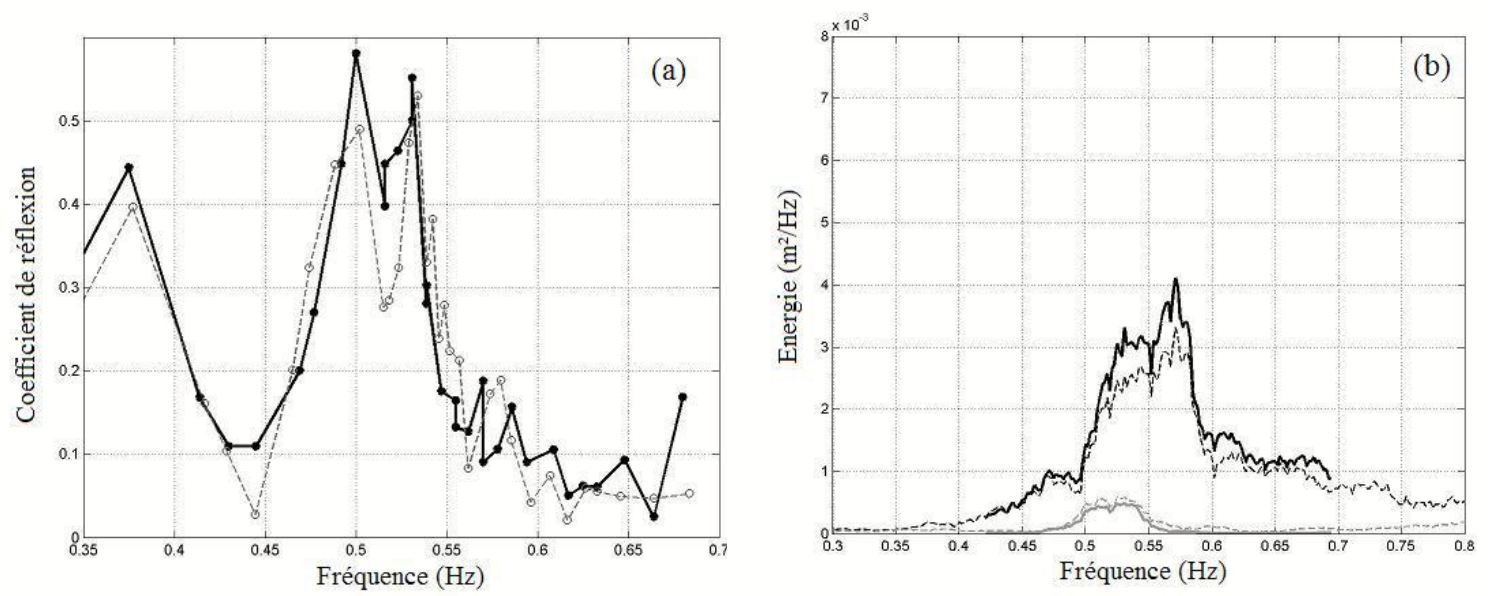

Fig. 1: (a) : Coefficient de réflexion calculé à partir des données des sondes à houle (tiret) et de l'ADV (trait plein) avec courant.

(b): Evolution de l'énergie pour une houle spectrale de période $\mathrm{T}_{\mathrm{p}}=1.813 \mathrm{~s}$ à partir des données des sondes à houle (trait plein) et de l'ADV (tiret) avec courant, dans l'hypothèse d'une onde partiellement stationnaire: onde incidente (noir) et onde réfléchie (gris).

Pour une houle irrégulière (de type Jonswap) de période $\mathrm{Tp}=1.813 \mathrm{~s}(\mathrm{f}=0.552 \mathrm{~Hz})$, les spectres d'énergies obtenus à partir des données des sondes à houle et de l'ADV dans l'hypothèse d'une onde partiellement stationnaire sont présentés sur la figure 1(b).

On observe une réflexion de $10.74 \%$ en énergie autour de $T_{p}$, ce qui correspond à un coefficient de réflexion de $\mathrm{R}=32.6 \%$ en amplitude pour cette gamme de fréquence pour les vagues régulières. La comparaison des ondes incidente et réfléchie calculées à partir des données des sondes à houle et de l'ADV sont en bon accord excepté une sur-estimation de l'amplitude de l'onde incidente autour de la fréquence pic avec les sondes. Les résultats à partir des vitesses sont cependant tout à fait corrects, la méthode à trois sondes n'étant pas une référence absolue pour la valeur des coefficients de réflexion.

\section{Applications in situ}

\subsection{Influence de la profondeur d'immersion de l'ADV}

Durant trois jours (du 22 au 24 juin 2005), des expériences ont été effectuées sur la plage du Truc Vert (Gironde) pour étudier l'écoulement turbulent sous la surface libre en zone de déferlement. Pour collecter les données quatre ADV ont été disposés sur une ligne verticale à $0,13 \mathrm{~m}, 0,73 \mathrm{~m}, 1,32 \mathrm{~m}$ et 2,05 $\mathrm{m}$ au-dessus $\mathrm{du}$ fond dans la zone intertidale. Au début le système n'était pas immergé, mais progressivement le niveau d'eau a augmenté pour atteindre une profondeur maximum de $2,5 \mathrm{~m}$ à marée haute au niveau de l'instrumentation. Dans cette partie, nous nous intéressons à l'influence de la profondeur d'immersion des ADV pour la mesure des coefficients de réflexion selon deux méthodes différentes, 
l'utilisation des vitesses horizontale et verticale $\left(\mathrm{u}_{\mathrm{h}}, \mathrm{w}\right)$ ou de la vitesse horizontale et de la pression $\left(\mathrm{u}_{\mathrm{h}}, \mathrm{p}\right)$, et pour deux profondeurs d'immersion différentes (ADV $\mathrm{A} 2$ à $0,73 \mathrm{~m}$ et $\mathrm{A} 3$ à $1,32 \mathrm{~m}$ au dessus du fond). Un courant de $0,2 \mathrm{~m} / \mathrm{s}$ a été observé durant les mesures, et son influence s'est avérée négligeable sur les résultats pour les périodes de vagues considérées $(T>4 s)$.

La figure 2 présente le coefficient de réflexion, pour une onde se propageant à une fréquence de $\mathrm{f}_{\mathrm{p}}=0.11 \mathrm{~Hz}$ (changeant légèrement avec la marée), calculé à partir des données des deux ADV A2 et A3 suivant les deux méthodes $\left(u_{h}, w\right)$ et $\left(u_{h}, p\right)$.

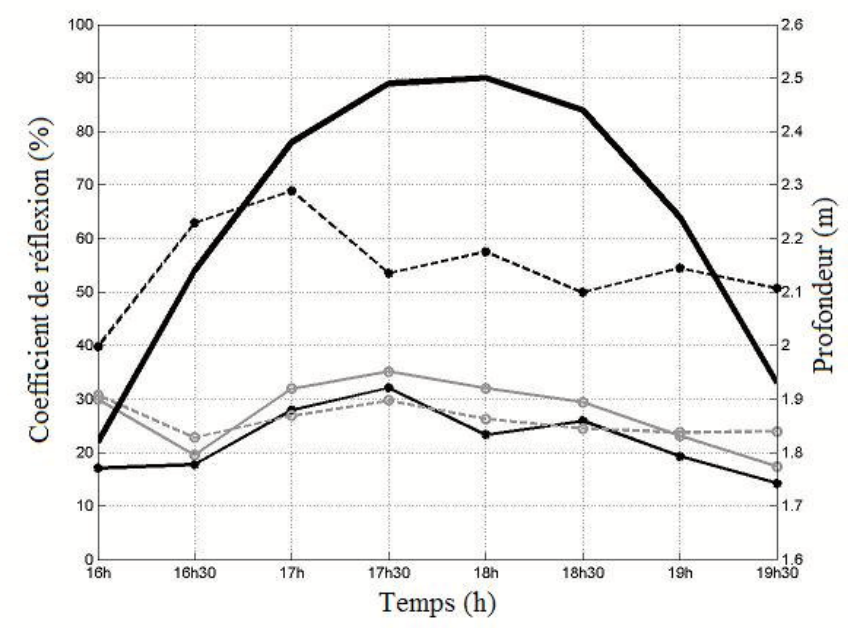

Fig. 2 : Coefficient de réflexion calculé à partir des deux méthodes $\left(u_{h}, p\right)$ (trait plein) et $\left(\mathrm{u}_{\mathrm{h}}, \mathrm{w}\right)$ (tiret) pour les deux ADV A2 (noir) et A3 (gris) avec l'évolution de la hauteur d'eau (trait plein noir en gras).

On observe une bonne concordance des résultats excepté avec la méthode $\left(\mathrm{u}_{\mathrm{h}}, \mathrm{w}\right)$ pour l'ADV A2. Ce dernier était placé assez près du fond et par conséquent la vitesse verticale est plus faible que proche de la surface libre, et donc beaucoup bruitée.

Nous pouvons conclure que pour des immersions des appareils de mesures près du fond, et particulièrement dans les eaux peu profondes, la méthode $\left(u_{h}, p\right)$ sera préconisée.

\subsection{Application dans la zone de déferlement}

Durant un mois (Novembre 2000), des expériences ont été effectuées sur la plage de Sète (Hérault) afin d'étudier l'action des vagues sur la formation de barres sédimentaires $^{2}$ et le rôle de ces barres dans le processus dissipatif dans la zone de déferlement ${ }^{3}$. Pour cela, trois courantomètres S4 ont été déployés sur la barre interne, la barre externe et le glacis (correspondant respectivement à une profondeur de 2,5 m (H1), $4 \mathrm{~m}(\mathrm{H} 2)$ et $6 \mathrm{~m}(\mathrm{H} 3))$. Des détails sur l'expérience sont présentés dans Certain ${ }^{4}$.

Ici, nous nous focalisons sur l'étude de l'état de mer lors d'une tempête puis résultant de la superposition d'une houle et d'une mer du vent. 
Pour la tempête du 12 novembre 2000, les spectres d'énergie calculés à partir des données de vitesses et de pression présentent un pic, de fréquence $0.13 \mathrm{~Hz}$ pour les trois $\mathrm{S} 4 \mathrm{H} 1, \mathrm{H} 2$ et $\mathrm{H} 3$ (Fig. 3).
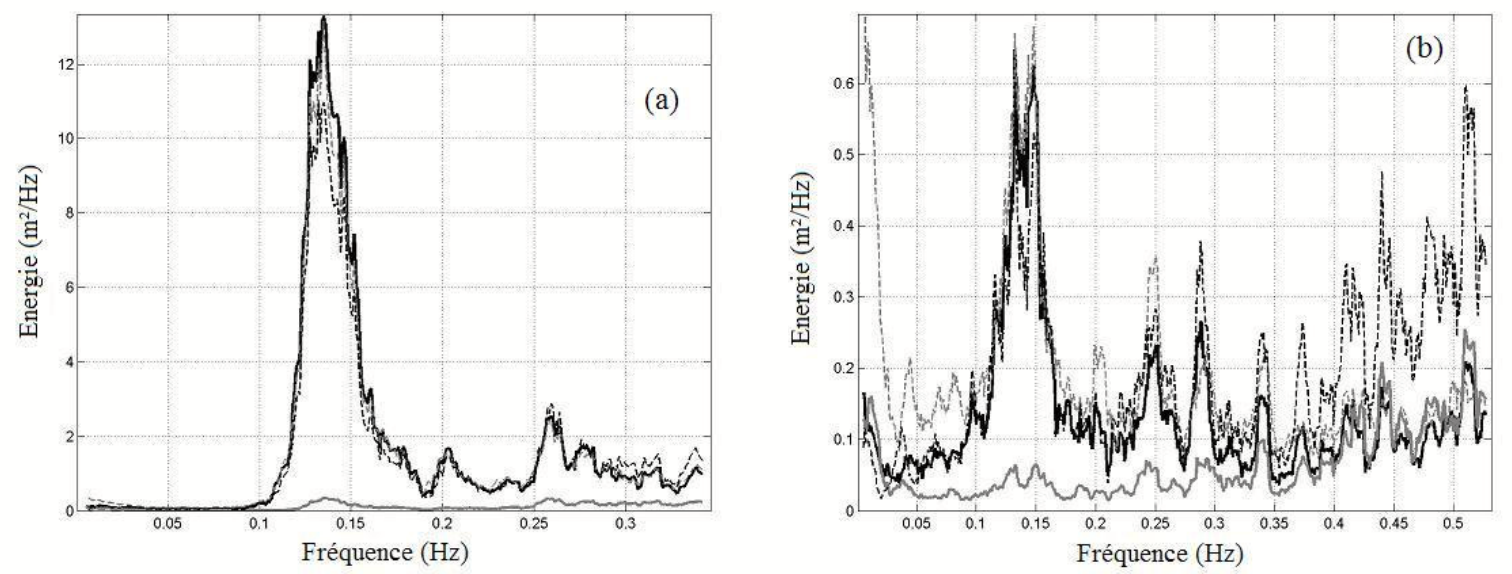

Fig. 3 : Spectres d'énergie pour les deux S4 disposés sur le glacis (a) et la barre interne (b) : dans l'hypothèse d'une onde progressive avec la vitesse horizontale (tiret gris) et la pression (tiret noir) ; et dans l'hypothèse d'une onde partiellement stationnaire : onde incidente (noir) et onde réfléchie (gris).

On observe une diminution progressive de l'énergie du S4 H3 à H1 ce qui indique que l'onde déferle sur la barre externe. Cependant, même après déferlement (au niveau de la barre interne (S4 H1)), on retrouve les caractéristiques de l'onde incidente avec un pic de fréquence de $0.13 \mathrm{~Hz}$ malgré un signal bruité. La réflexion est relativement faible, le processus dissipatif est alors prédominant.

La figure 4 montre le spectre d'énergie calculé à partir des données des deux S4 $\mathrm{H} 1$ et $\mathrm{H} 3$ avec les deux hypothèses onde progressive et onde partiellement stationnaire pour la superposition d'une houle et d'une mer du vent.
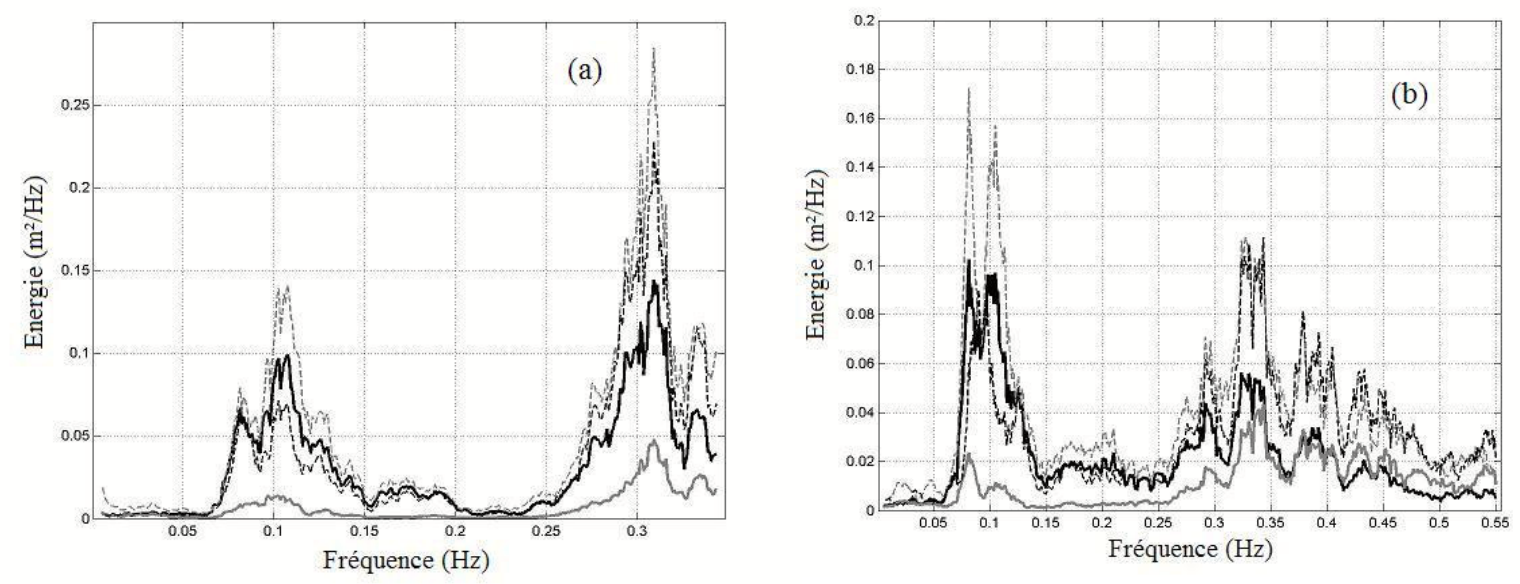

Fig. 4 : Spectre d'énergie pour les deux S4 disposés sur le glacis (a) et la barre interne (b): dans l'hypothèse d'une onde progressive avec la vitesse horizontale (tiret gris) et la pression (tiret noir); et dans l'hypothèse d'une onde partiellement stationnaire: onde incidente (trait plein noir) et onde réfléchie (trait plein gris). 
Nous observons deux pics. Le premier, situé autour de $\mathrm{f}_{\mathrm{p}}=0.1 \mathrm{~Hz}$ correspond à une vague de longue période, et le second, situé autour de $\mathrm{f}_{\mathrm{p}}=0.31 \mathrm{~Hz}$, correspond aux vagues de vent. Pour ces deux ondes, l'hypothèse d'onde progressive est représentative mais l'interprétation de ces résultats n'est pas forcément évidente. En effet, les spectres identiques obtenus à partir de la vitesse horizontale et de la pression pour la mer du vent laissent à penser que la réflexion est faible. En fait, les vagues de vent déferlent en partie avant la fosse interne (l'énergie de l'onde incidente passe de 0.14 à $0.05 \mathrm{~m}^{2} / \mathrm{Hz}$ ), la réflexion observée au niveau du glacis est à peu près identique en énergie à celle mesurée au niveau de la fosse interne. Le coefficient de réflexion est total au niveau de la fosse interne, ce qui semble correspondre à une réflexion par le haut de plage.

\section{Discussion et conclusion}

La mesure de vagues en eau profonde et intermédiaire a été largement étudiée, notamment l'estimation du spectre directionnel ${ }^{1}$. Notre étude se porte sur la mesure d'onde partiellement stationnaire en prenant en compte l'influence $d u$ courant et la direction de propagation. De plus en plus d'instruments sophistiqués basés sur la mesure de l'accélération de la surface libre, la variation de pression ou les vitesses dans le fluide ont prouvé leur efficacité. L'information sur le caractère partiellement stationnaire de la houle présente un intérêt particulier pour l'étude de plages réflectives ou d'agitation au voisinage de structures.

Ce papier montre que l'information sur le caractère partiellement stationnaire d'une houle peut être facilement mesuré au moyen de données synchrones de vitesses horizontale et verticale ou de pression. Ces deux méthodes peuvent être utilisées lorsque les instruments sont déployés assez loin du fond, typiquement en eau profonde où le mouvement induit par la vague n'affecte que la couche supérieure $(z<\lambda / 2)$. En zone côtière, les mesures sont souvent effectuées au voisinage $\mathrm{du}$ fond, on utilisera alors des données synchrones de vitesse horizontale et de pression.

Ces méthodes restent valides en présence d'un courant uniforme. Nous avons observé cependant que l'influence du courant reste limitée. Les expériences récentes ont prouvé que l'onde semble être beaucoup plus sensible au courant moyen qu'à ses fluctuations locales ${ }^{6}$.

\section{Remerciements.}

Les auteurs remercient le Conseil Général du Var pour son support financier pour les expériences menées au BGO FIRST dans le cadre du GIS HYDRO. Les expériences à Sète et sur la plage du Truc Vert ont été menées respectivement dans le cadre du programme scientifique PNEC (dynamique de plages à barres) et du PATOM (hydrodynamique dans la zone affectée par le déferlement), leur support financier et les participants sont remerciés. 


\section{Références}

1 Benoit M., Frigaard P.and Schäer H.A., (1997), Analyzing multidirectional wave spectra: a tentative classification of available methods, in proceedings of the 1997 IAHR conference, San Fransisco, pp. 131-158, the Johns Hopkins university press, Baltimore.

2 Certain R., Barusseau J. P., Capobianco R., Meuret A., Rey V., Dulou C., Stepanian A., Levoy F. and Howa H., (2001), Bottom and shoreline evolutions under wave actions at a french Mediterranean site : The beach of Sète, The fifth International Conference on the Mediterranean Coastal Environment, 23-27 October 2001, Hamammet, Tunisia

3 Certain R., Meule S., Rey V. and Pinazo C., (2005), Swell transformation on a microtidal barred beach (Sète, France), Journal of Marine Systems, 38, 19-34.

4 Certain R., (2002), Morphodynamique d'une côte sableuse microtidale à barres: le golfe du Lion (Languedoc-Roussillon), Thèse de doctorat, Univ. Perpignan, 209 p.

5 Drevard D., Meuret A., Rey V., Piazzola J. and Dolle A., (2003), Partially reflected waves measurements using Acoustic Doppler Velocimeter (ADV), 13th International Offshore and Polar Engineering Conference ISOPE'03, Honolulu, Hawai, 25-31 may 2003.

6 Magne R., Rey V. and Ardhuin F., (2005), Measurement of wave scattering by topography in presence of currents, Physics of Fluids, in press.

7 Rey V., Capobianco R. and Dulou C., (2002), Wave scattering by a submerged plate in presence of a steady uniform current, Coastal Engineering, 47(1), 27-34.

8 Walton Jr., T., L., (1992), Wave reflection from natural beaches, Ocean Engineering, 19(3), 239-258.

9 Wright L.D. and Short A. D., (1984), Morphodynamic variability of surf zone and beaches: a synthesis, Marine Geology, 56, 93-118. 